\title{
Context Inconsistency Elimination Based on User Feedback and Modified Evidence Theory
}

\author{
Leitao Wang, Hongji Xu, Guoxia Sun, Zhengfeng Du, Zhigang Xie, and Lina Zheng
}

\begin{abstract}
In dynamic and open environment, context aware system obtains context information from the dynamic, distributed and heterogeneous sources, but the context information usually has inconsistencies which would lead to inappropriate services. We proposed a new context inconsistency elimination algorithm based on user feedback and modified evidence theory in this paper. Through user feedback, each sensor's perception precision can be acquired, and with the modified evidence theory, we can make full use of all context information and eliminate inconsistent context by adjusting the influence of every context on whole judgment based on sensor perception precision. In order to evaluate the performance of the proposed context inconsistency elimination algorithm, context aware rate is defined. The experiment results show that the proposed context inconsistency elimination algorithm can obtain the best context aware rate in most cases when the error rates of sensors are varied.
\end{abstract}

Index Terms-Adaptive service, context aware, context inconsistency elimination, user feedback

\section{INTRODUCTION}

Context aware technology is one of the key technologies of ubiquitous computing [1], [2]. Various sensors can be applied to deriving context. Through the consideration of contexts, the context aware systems are able to adapt their services to the continuously evolving situations without diverting human attention so as to improve the quality of human-computer interaction experience.

However, due to the precision differences of sensors, equipment isomerism and network delay, the context aware information usually has inconsistencies [3]. Thus, we should eliminate those inconsistencies so as to obtain the correct context information [4]. Otherwise the services we provide would be inappropriate.

In [5], the authors present the trust-worthiness based algorithm which selects the context information with the largest sensor perception precision among all context information. In [6] the voting algorithm is applied to select the context information by plurality. In addition, efficient context aware selection based on user feedback algorithm which takes

Manuscript received September 20, 2013; revised November 18, 2013. This work is supported by China Postdoctoral Science Foundation funded project (2011M501092), Special Fund for Postdoctoral Innovative Projects of Shandong Province (201103003), Scientific Research Foundation for the Excellent Young and Middle-aged Scientists of Shandong Province (BS2012DX024), Open Research Fund of State Key Lab. of Mobile Communications (2011D08), Independent Innovation Foundation of Shandong University (2012ZD035, 2012TS082).

The authors are with School of Information Science and Engineering, Shandong University, Jinan, China (e-mail: hongjixu@sdu.edu.cn, zfdu@sdu.edu.cn). trust-worthiness based algorithm [5] and voting based algorithm [6] into consideration is proposed in [7]. In this algorithm, the authors set accepted threshold and rejection threshold. If there is a sensor perception precision which is larger than accepted threshold, the service provider will select the context information of this sensor as final output; or if the sensor perception precisions of all sensors are under the rejection threshold, the trust-worthiness based algorithm will be used; otherwise, voting algorithm would be applied. Nevertheless, above mentioned algorithms cannot make full use of all context information, so they could not obtain outstanding performance.

In [8]-[10], the parameter of Quality of Context (QoC) in middleware is used to manage context information. In [11], QoC is measured on context source layer, context processor layer and context consumer layer. In their work, context inconsistency would be eliminated based on one QoC parameter directly or the combination of several QoC parameters. But just through the utilization of QoC, the inconsistent context cannot be eliminated effectively. Except that, the maneuverability of their methods is also restricted.

Furthermore, the authors of [12] propose an approach to detect problematic contexts and resolve the context inconsistencies with the help of context aware application level semantics. However, it does not support real-time requirements and the error recovery would cause extra resource consumption.

In this paper, we present a new context inconsistency elimination algorithm based on user feedback and modified evidence theory. It can make full use of all context information and obtain a better performance.

The rest of the paper is organized as follows. Section II describes the proposed algorithm which is used to eliminate the inconsistent context. Section III presents the performance evaluation and experiment results. Finally we draw the conclusion in Section IV.

\section{CONTEXT INCONSISTENCY ELIMINATION BASED ON USER FEEDBACK AND MODIFIED EVIDENCE THEORY}

Considering smart home as a typical application scenario, various sensors are applied to deriving context.

We can acquire user feedback data from various statuses of home appliances and sensors attached to them such as on/off button or remote controller. Based on the data of user feedback, we can estimate each sensor's perception precision. For example, if the context aware system recognizes the user location context as "meeting room", the system would turn on the projector of the meeting room automatically. After a while the user operates the lamp at kitchen, and the user feedback 
indicates the real situation as "kitchen", but the system perhaps wrongly recognizes the user location context as "meeting room". In this case, the system would demonstrate that the current sensor perception has failed. Whenever user feedback data is acquired, an evaluation of sensor's perception precision would be performed. With the modified evidence theory proposed by this paper, we can make full use of all context information by adjusting the influence of every context on whole judgment based on sensor perception precision. Finally, using Dempster's basic reliability synthesis principle [13], the inconsistency of context information will be eliminated.

The procedure of proposed context inconsistency elimination algorithm is shown in Fig. 1, and the processing steps are explained as follows.

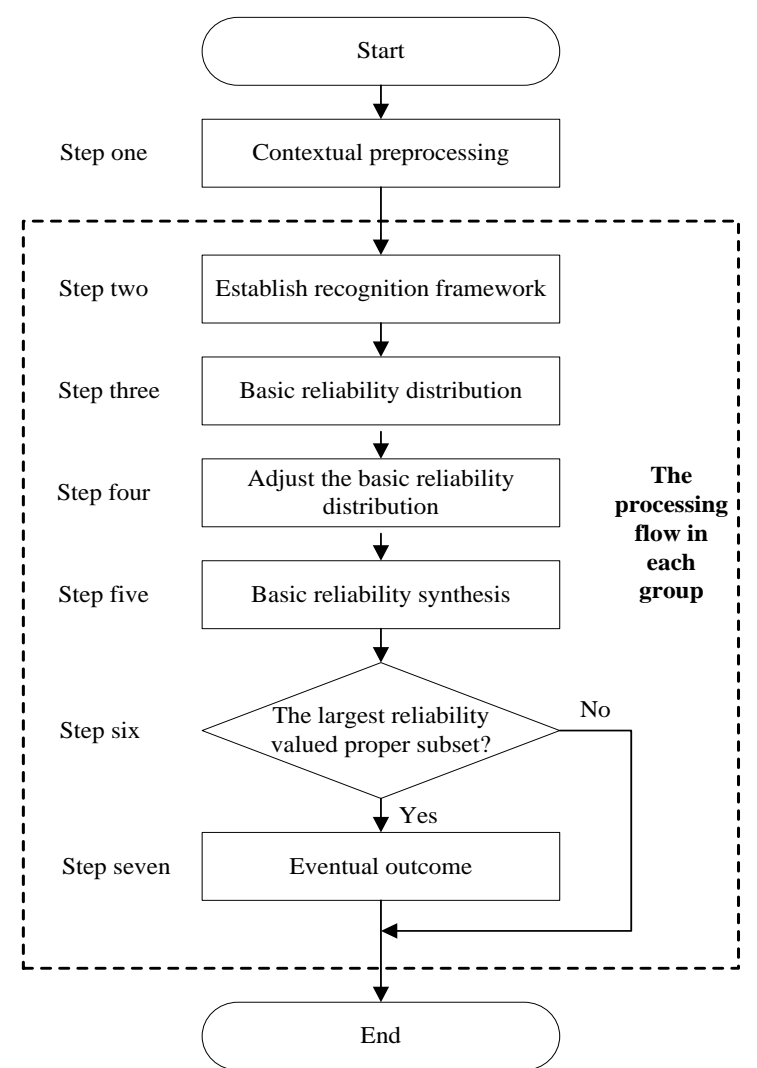

Fig. 1. The procedure of proposed context inconsistency elimination algorithm.

\section{A. Step One: Contextual Preprocessing}

In this step, we collect context information captured by all kinds of sensors, model the context information according to the context modeling mode [14], and then classify the context information according to the context awareness type. The context modeling mode is "awareness type + awareness information + awareness precision".

By classifying the context information according to the context awareness type, context inconsistency elimination can be divided into synchronous processing parts so as to make full use of computing resources, which can improve the real-time performance of context inconsistency elimination.

Step two to Step seven are discussed in one context group.

\section{B. Step Two: Establish Recognition Framework}

We use Dempster-Shafer evidence theory [13] to establish recognition framework. In Dempster-Shafer evidence theory, there are four definitions.

Definition 1: The recognition framework $\Theta$ is defined as an exhaustive set of mutually exclusive events or propositions of a particular experiment.

Definition 2: If for any subset $A$ of recognition framework $\Theta$, it satisfies $m(\phi)=0, \sum_{A \subset \Theta} m(A)=1$, then $m$ will be called the basic reliability distribution function of recognition framework $\Theta . \forall A \subset \Theta, m(A)$ is the basic reliability value of subset $A$. If $m(A)>0$, then $A$ is the focal element of the recognition framework $\Theta$.

Definition 3: For recognition framework $\Theta$, the degree of belief about subset $X$ is defined as

$$
\operatorname{Bel}(X)=\sum_{Y \subseteq X} m(Y)
$$

where $X$ and $Y$ are subsets of recognition framework $\Theta$.

Definition 4: Assume $m_{1}$ and $m_{2}$ are basic reliability distribution function on the same recognition framework. $A_{1}, A_{2}, \ldots, A_{k}$ are focal elements of $m_{1}$ and $B_{1}, B_{2}, \ldots, B_{k}$ are focal elements of $m_{2}$. Then Dempster's basic reliability synthesis principle can be expressed as

$$
m(C)=\frac{\sum_{A_{i} \cap B_{j}=A} m_{l}\left(A_{i}\right) m_{2}\left(B_{j}\right)}{1-K}
$$

$$
\text { Subject to: } K=\sum_{A_{i} \cap B_{j}=\phi} m_{1}\left(A_{i}\right) m_{2}\left(B_{j}\right)
$$

Dempster's basic reliability synthesis principle can be directly extended to $N$ sensors.

In one context group, recognition framework is the exhaustive set of mutually exclusive results apperceived by every sensor.

\section{Step Three: Basic Reliability Distribution}

Each sensor's sensing result in one time can be considered as a basic reliability distribution function on recognition framework and this basic reliability distribution function can be expressed as

$$
m(A)=\left\{\begin{array}{l}
1, A=\text { subset_awared } \\
0, \text { A } \neq \text { subset_awared }
\end{array}\right.
$$

where subset_awared is the focal element of the recognition framework perceived by this sensor.

For each sensor, the focal elements of the recognition framework $\Theta$ are different. In other words, each sensor's sensing result may be inconsistent, so it is hard to make a final decision and we must take context inconsistency elimination algorithm into consideration.

\section{Step Four: Adjust the Basic Reliability Distribution}

We can acquire user feedback data from various statuses of home appliances and sensors attached to them. Based on the 
data of user feedback, we can estimate each sensor's perception precision. The sensor perception precision $r$ can be defined as

$$
r=1-\frac{\text { Mismath }_{u f}}{\text { Total }_{u f}}
$$

where Mismatch $_{u f}$ is the number of mismatches between sensors' perception context and user feedback data, and Total $_{u f}$ is the total number of user feedback data. If there have no user feedback data in one time, we will use the last estimated sensor perception precision as the sensor perception precision of now.

Based on evidence theory, we proposed a novel algorithm which been called modified evidence theory. In this algorithm, we use (5) to readjust the basic reliability distribution function

$$
m_{r}(A)= \begin{cases}r^{n} \cdot m(A) & A \subset \Theta \\ 1-\sum_{B \subseteq \Theta} m(B) & A=\Theta\end{cases}
$$

where $r$ is sensor perception precision, $n$ is the exponent of $r$ and $n$ been called weighting coefficient which can be adjusted according to the dynamic conditions.

\section{E. Step Five: Basic Reliability Synthesis}

Using Dempster's basic reliability synthesis principle [13] and (1), (5), we can calculate the belief degree about every subset of recognition framework.

\section{F. Step Six: Select the Proper Subset Which Has the Largest Value of Belief Degree}

In this step, every proper subset's belief degree value will be compared. If a subset has not the largest value of belief degree or it is not the proper subset, this subset will be neglected. On the contrary, if a proper subset has the largest value of belief degree, the content of this proper subject will be output as the final result.

\section{G. Step Seven: Eventual Outcome}

We choose the content of proper subset which be selected by step six as the eventual outcome.

For one context awareness type, there have many sensors and each sensor's sensing result may be inconsistent, so we must take context inconsistency elimination algorithm into consideration. Through the seven steps of proposed context inconsistency elimination algorithm, we can pick out one proper subset which has the largest value of belief degree from the recognition framework. Because this proper subset has only one element and the content of this subject will be chose as the final output, we eliminate the inconsistency of context information.

\section{The PERFORMANCE EVALUATION}

In the experiment, we compare the performance of several context inconsistency elimination algorithms including our proposed algorithm, the trust-worthiness based algorithm [5], the voting based algorithm [6], the efficient context aware selection based on user feedback algorithm [7].

In order to evaluate the performance of different context inconsistency elimination algorithms, we define the context aware rate [7] as follows

$$
C_{a r}=\frac{\text { Corret }_{\text {context }}}{\text { Total }_{\text {context }}}
$$

where Corret $_{\text {context }}$ is the total number of correct context, and Total $_{\text {context }}$ is the total number of context occurrence.

Table I shows the abbreviations used for context inconsistency elimination.

TABLE I: THE ABBREVIATIONS USED FOR CONTEXT INCONSISTENCY ELIMINATION

\begin{tabular}{ll}
\hline \hline \multicolumn{1}{c}{ Symbols } & Meaning \\
\hline A-trust & The trust-worthiness based algorithm \\
A-vote & The voting based algorithm \\
$A-f b$ & The efficient context aware selection based on user \\
& feedback algorithm \\
$A-d s-f d$ & Proposed algorithm \\
$r_{i}$ & Perception precision of sensor $S_{i}$ \\
$E_{i}$ & $1-r_{i}$ i.e. Perception error rate of sensor $S_{i}$ \\
\hline \hline
\end{tabular}

In order to get the user feedback data and evaluate the performance of context aware rate achieved by proposed algorithms, ten human subjects and five sensors are participated in the experiment. The number of context occurrences is set to be 40,000 .

In order to illustrate the influence of the weighting coefficient on performance, we evaluate the context aware rate of the proposed context inconsistency elimination algorithm with respect to various weighting coefficients. In this experiment six groups of sensors are applied. In each group, five sensors $S_{1}, S_{2}, S_{3}, S_{4}, S_{5}$ are participated in the experiment and the perception error rates of five sensors are changeless within each experiment.

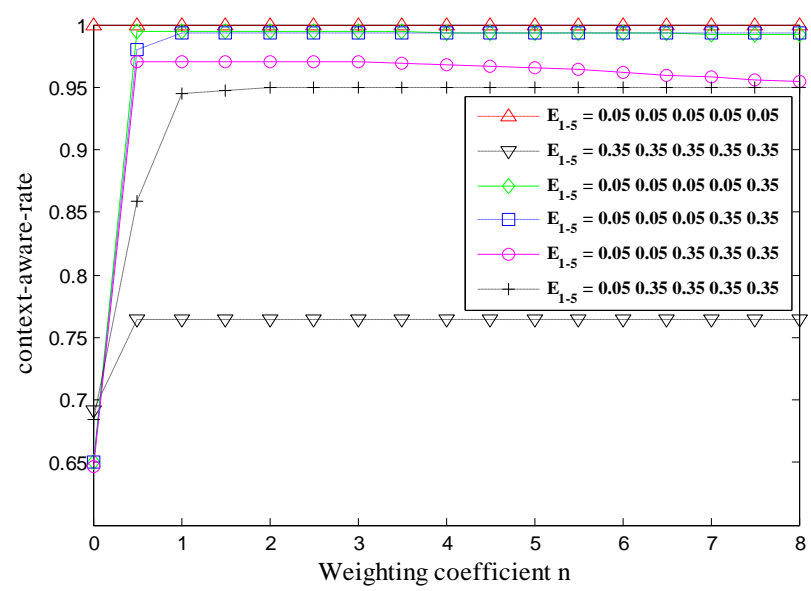

Fig. 2. The $C_{a r}$ with respect to $n$.

Fig. 2 illustrates the context aware rate of the proposed context inconsistency elimination algorithm with respect to various weighting coefficients. The numerical values in the legend of Fig. 2 are perception error rates of five sensors 
$S_{1}, S_{2}, S_{3}, S_{4}, S_{5}$ respectively. From Fig. 2, we can see that the performance of the proposed algorithm is superior when the weighting coefficient is 2 . When the weighting coefficient is smaller than 2, particularly smaller than 0.5 , the performance of the proposed algorithm drops rapidly. At the same time, when the weighting coefficient is larger than 2 , there will be a slow drop of the performance. In the following experiment, we set the weighting coefficient as 2 so as to achieve the best performance.

In order to evaluate the effectiveness of our proposed context inconsistency elimination algorithm, five sensors $S_{1}, S_{2}, S_{3}, S_{4}, S_{5}$ are participated in the experiment. It is assumed that the perception error rates of sensors $S_{2}, S_{3}, S_{4}, S_{5}$ are changeless within each experiment, but the perception error rates of sensor $S_{1}$ vary from small to large.

Fig. 3 to Fig. 7 illustrate the context aware rate of the proposed context inconsistency elimination algorithm with the weighting coefficient equal to 2 . In each experiment, we compare the performance of several context inconsistency elimination algorithms including our proposed algorithm, the trust-worthiness based algorithm [5], the voting based algorithm [6], the efficient context aware selection based on user feedback algorithm [7].

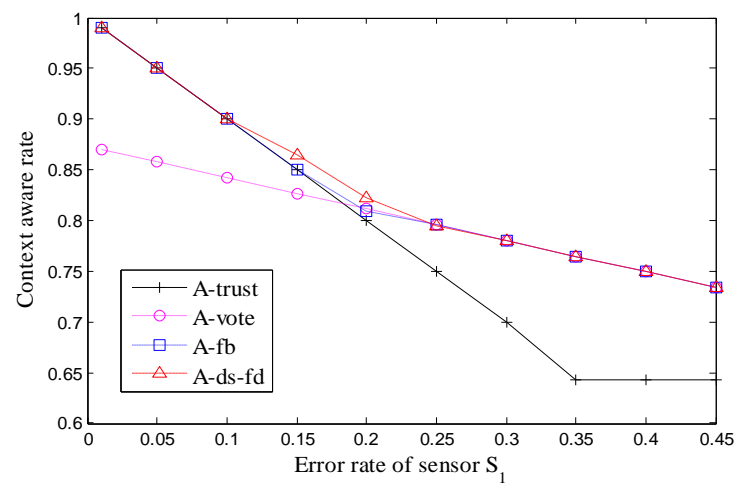

Fig. 3. The $C_{a r}$ with respect to error rate $E_{1}$.

$\left(E_{2}=0.35 E_{3}=0.35 E_{4}=0.35 E_{5}=0.35\right)$

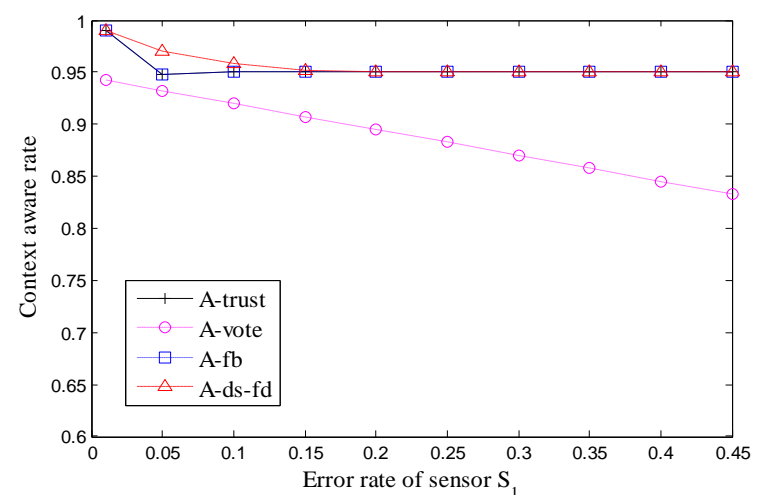

Fig. 4. The $C_{a r}$ with respect to error rate $E_{1}$.

$\left(E_{2}=0.05 E_{3}=0.35 E_{4}=0.35 E_{5}=0.35\right)$

The context aware rate of the four algorithms with $E_{2}=0.35, E_{3}=0.35, E_{4}=0.35, E_{5}=0.35$ and different error rates $E_{1}$ of $S_{1}$ is shown in Fig. 3. When the error rate $E_{1}$ is smaller than $10 \%$, the performance of
$A_{-} d s_{-} f d$ is equal to $A_{-} f b$ and $A_{-}$trust , in the meantime it is better than $A_{-}$vote. However, when the error rate of $E_{1}$ is larger than $25 \%$, the performance of $A_{-} d s_{-} f d$ is equal to $A_{-} f b$ and $A_{-}$vote, and meantime it is better than A_trust. In addition, $A \_d s_{-} f d$ obtains a higher context aware rate when the error rate $E_{1}$ is between $10 \%$ and $25 \%$.

Fig. 4 illustrates the context aware rate of the four algorithms with $E_{2}=0.05, E_{3}=0.35, E_{4}=0.35, E_{5}=0.35$ and different error rates $E_{1}$ of $S_{1}$. We can see that $A \_d s_{-} f d$ obtains a higher context aware rate obviously when the error rate $E_{1}$ is smaller than $20 \%$. At the same time, when the error rate $E_{1}$ is larger than $20 \%$, the performance of $A_{-} d s_{-} f d$ is equal to $A_{-} f b$ and $A_{-}$trust, moreover it is better than $A_{-}$vote

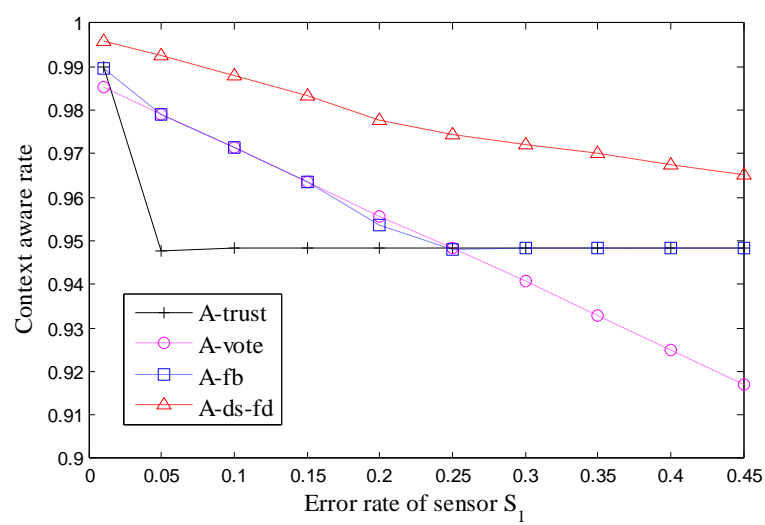

Fig. 5. The $C_{a r}$ with respect to error rate $E_{1}$ $\left(E_{2}=0.05 E_{3}=0.05 E_{4}=0.35 E_{5}=0.35\right)$

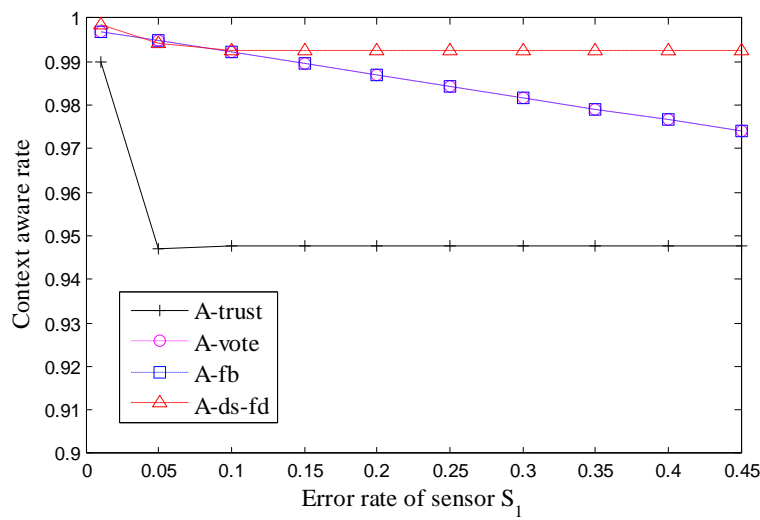

Fig. 6. The $C_{a r}$ with respect to error rate $E_{1}$. $\left(E_{2}=0.05 E_{3}=0.05 E_{4}=0.05 E_{5}=0.35\right)$

Fig. 5 demonstrates the context aware rate of the four algorithms with $E_{2}=0.05, E_{3}=0.05, E_{4}=0.35$, $E_{5}=0.35$ and different error rates $E_{1}$ of $S_{1}$. It shows that $A \_d s_{-} f d$ obtains significantly higher context aware rate than other three algorithms when the error rate $E_{1}$ are varied.

In Fig. 6, the context aware rate of the four algorithms with $E_{2}=0.05, E_{3}=0.05, E_{4}=0.05, E_{5}=0.35$ and different error rate $E_{1}$ of $S_{1}$ is compared. When the error rate $E_{1}$ is smaller than $10 \%$, the performance of $A_{-} d s_{-} f d$ is similar to 
$A_{-} f b$ and A_vote, in the meantime it is larger than A_trust . When the error rate $E_{1}$ is larger than $10 \%$, the performance of $A_{-} d s_{-} f d$ is better than $A_{-} f b, A_{-}$trust and A_vote.

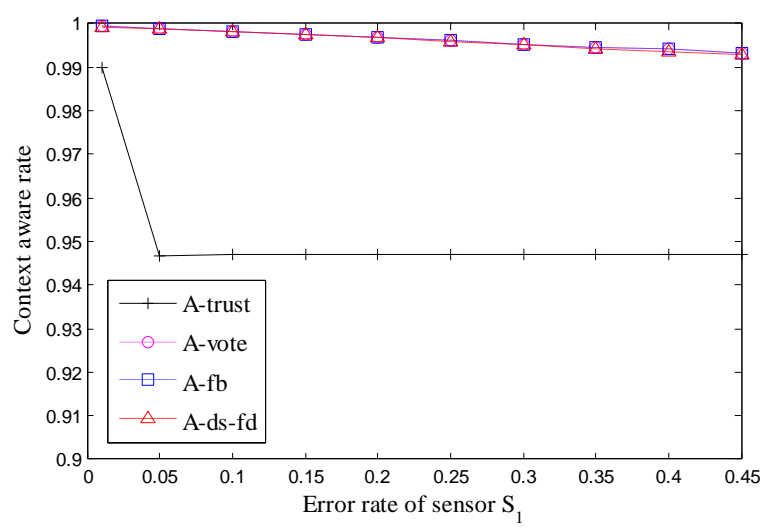

Fig. 7. The $C_{a r}$ with respect to error rate $E_{1}$. $\left(E_{2}=0.05 E_{3}=0.05 E_{4}=0.05 E_{5}=0.05\right)$

Fig. 7 shows the context aware rate of the four algorithms with $E_{2}=0.05, E_{3}=0.05, E_{4}=0.05, E_{5}=0.05$ and different error rates $E_{1}$ of $S_{1}$. The performance of $A \_d s_{-} f d$ is similar to $A_{-} f b$ and $A_{-}$vote, but it is larger than $A_{-}$trust distinctly when error rate $E_{1}$ are varied.

Through analyzing of Fig. 3 to Fig. 7, we find that the proposed context inconsistency elimination algorithm $A \_d s_{-} f d$ can obtain the best context aware rate in most cases compared to the other algorithms. For instance, in Fig.4, the experiment results show that the proposed context inconsistency elimination algorithm improves the context aware rate by up to $3.9 \%$ compared to the trust-worthiness based method, $2.2 \%$ compared to the voting method and $2.4 \%$ compared to the efficient context-aware selection based on user feedback method when the error rate $E_{1}$ is equal to 0.2 .

\section{CONCLUSION}

In this paper, we presented a new context inconsistency elimination algorithm based on user feedback and modified evidence theory. By classifying the context information according to the context awareness type and building each group's recognition framework respectively, context inconsistency elimination can be divided into synchronous processing parts so as to make full use of computing resources, which can improve the real-time performance of context inconsistency elimination. Through user feedback, we can get each sensor's perception precision. Based on sensors' perception precision and modified evidence theory algorithm, we can make full use of all context information and eliminate inconsistent context by adjusting the influence of every context on whole judgment.

The experiment results show that the proposed context inconsistency elimination algorithm $A_{-} d s_{-} f d$ obtains the best context aware rate in most cases compared to A_trust, $A_{-}$vote and $A_{-} f b$ algorithms when the error rates of the sensors are varied.

\section{REFERENCES}

[1] A. Amnal, A. Dewabharata, S. Y. Chou, and M. Erawan, "Activity modeling using semantic-based reasoning to provide meaningfu context in human activity recognizing," in Proc. Institute of Industrial Engineers Asian Conference 2013, Singapore, 2013, pp. 1081-1089.

[2] I. Satoh, "Reusable context-aware software," in Proc. 7th International Conference on Ubiquitous Information Technologies and Applications, Hong Kong, 2013, pp. 251-259.

[3] C. Xu, Y. Liu, S. C. Cheung, C. Cao, and J. Lv, "Towards context consistency by concurrent checking for internetware applications," The Journal of Science China Information Sciences, vol. 56, no. 8, pp. 1-20, Aug. 2013

[4] C. Xu and S. C. Cheung, "Inconsistency detection and resolution for context-aware middleware support," in Proc. 13th ACM SIGSOFT International Symposium on Foundations of Software Engineering, New York, 2005, pp. 336-345.

[5] A. Manzoor, H. L. Truong, and S. Dustdar, "On the evaluation of quality of context," in Proc. 3rd European Conference on Smart Sensing and Context 2008, Zurich, 2008, pp. 140-153.

[6] D. F. McAllister, C. E. Sun, and M. A. Vouk, "Reliability of voting in fault-tolerant software systems for small output-spaces," IEEE Trans. Reliability, vol. 39, no. 5, pp. 524-534, Dec. 1990.

[7] B. H. Lee, and D. H. Kim, "Efficient context-aware selection based on user feedback," IEEE Trans. Consumer Electronics, vol. 58, no. 3, pp 978-984, Aug. 2012.

[8] A. Manzoor, H. L. Truong, and S. Dustdar, "Quality aware context information aggregation system for pervasive environments," in Proc. International Conference on Advanced Information Networking and Applications Workshops 2009, Bradford, 2009, pp. 266-271.

[9] H. Yan, D. Zheng, and J. Wang, "Research of quality based autonomic context processing for pervasive applications," in Proc. 7th International Conference on Computer Science \& Education, Melbourne, 2012, pp. 1234-1238.

[10] D. Zheng, Q. Xu and K. R. Ben, "Research of qoc-aware service adaptation in pervasive environment," in Proc. International Conference on Intelligent Computing, Huangshan, 2012, pp. 284-292.

[11] X. K. Yang, "An adaptive mechanism for inconsistent contex resolution in ubiquitous computing," in Proc. International Conference on Control Engineering and Communication Technology, Liaoning, 2012, pp. 703-706.

[12] C. H. Chen, C. Y. Ye, and H. A Jacobsen, "Hybrid context inconsistency resolution for context-aware services," in Proc. IEEE International Conference on Pervasive Computing and Communications, Seattle, 2011, pp. 10-19.

[13] G. Shafer, A Mathematical Theory of Evidence, Princeton, NJ: Princeton Univ. Press, 1976.

[14] X. Zhang, B. Hu, J. Chen, and P. Moore, "Ontology-based context modeling for emotion recognition in an intelligent web," The Journal of World Wide Web, vol. 16, no. 4, pp. 497-513, Sep. 2013.

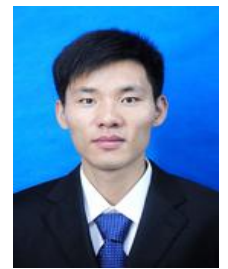

Leitao Wang received the B.S. degree in electronic information science and technology from Shandong Agricultural University, Taian, China in 2011. He is currently a M.S. student in the School of Information Science and Engineering at Shandong University, Jinan, China. His research interests include machine learning, data fusion, wireless sensor network and cloud computing.

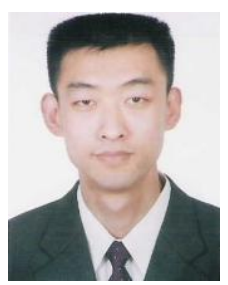

Hongji Xu received the B.S. degree in electronic engineering from Shandong University of Technology, Jinan, China in 1999, and received the M.S. degree in signal and information processing and the Ph.D. degree in communication and information system from Shandong University, Jinan, China, in 2001 and 2005 , respectively. He is currently an associate professor in the School of Information Science and Engineering at Shandong University. From 2004 to 2005, he was a visiting Ph.D. candidate at Telecommunications Technological Center of Catalonia (CTTC) and Department of Signal Theory and Communication, Polytechnic University of Catalonia (UPC), Barcelona, Spain and did research in the areas of wireless communication and signal processing. Since 2010 he has been a post-doctoral in Tsinghua University - Inspur Group post-doctoral scientific research station, China, and focused on research in multimedia information processing for smart 
home and cloud computing. His research interests include space-time processing for wireless communications, smart antenna, blind signal processing, ubiquitous computing and intelligent sensing.

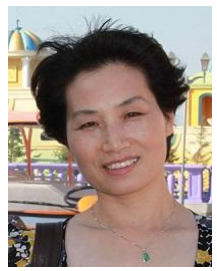

Guoxia Sun received the B.S. degree in electrical engineering, the M.S. degree in signal and information processing and the $\mathrm{Ph} . \mathrm{D}$. degree in communication and information system from Shandong University, Jinan, China in 1984, 1997 and 2007 respectively. Currently she is an associate professor in the School of Information Science and Engineering, Shandong processing, pattern recognition and biometrics.

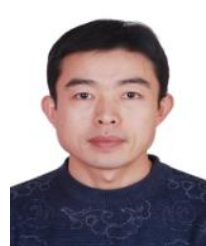

Zhengfeng Du received the B.S. degree in physics and the M.S. degree in electrical engineering from Shandong University, Jinan, China in 1998 and 2003, respectively, and the Ph.D. degree in electrical engineering from Southeast University, Nanjing, China, in 2009. Currently he is a lecturer with the School of Information Science and Engineering, Shandong University. His research interests include cooperative communications and space-time processing techniques in wireless communications.

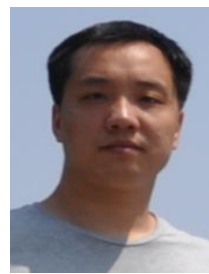

Zhigang Xie received the B.S. degree in electronic information science and technology from the PLA Information Engineering University, Zhengzhou, China in 2003, and received the M.S. degree in signal and information processing from Shandong University, Jinan, China in 2013. His research interests include machine learning, data fusion, pervasive computing and wireless sensor network.

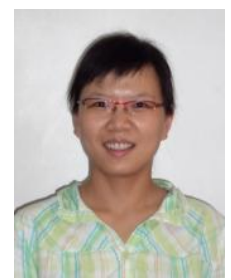

Lina Zheng received the B.S. degree in communication engineering from Shandong University, Jinan, China in 2001, the M.S. degree and the $\mathrm{Ph} . \mathrm{D}$. degree in communication and information system from Shandong University, Jinan, China, in 2004 and 2011, respectively. Her research interests include cross-layer design and routing algorithm in wireless ad hoc networks. 